\title{
Post-lockdown spread of COVID-19 from cities to vulnerable forest-fringe villages in Central India
}

\author{
Ruth DeFries ${ }^{1 *}$, Meghna Agarwala ${ }^{2}$, Sandra Baquie ${ }^{3}$, Pooja Choksi ${ }^{1}$, \\ Nitish Dogra ${ }^{4}$, G. S. Preetha ${ }^{4}$, Sarika Khanwilkar ${ }^{1}$, Pinki Mondal ${ }^{5}$, \\ Harini Nagendra ${ }^{6}$ and Johannes Urpelainen ${ }^{7}$ \\ ${ }^{1}$ Department of Ecology, Evolution, and Environmental Biology, 1200 Amsterdam Avenue, Columbia University, New York, NY 10027 \\ ${ }^{2}$ School of International and Public Affairs, 420 West $118 \mathrm{t}$ Street, New York, NY 10027 \\ ${ }^{3}$ Ashoka University, Rajiv Gandhi Education City, Sonipat 131 029, India \\ ${ }^{4}$ International Institute of Health Management Research, Plot No 3, Sector 18, Sector 18a Dwarka, Phase II, New De lhi, Delhi 110 075, India \\ ${ }^{5}$ Department of Geography and Spatial Sciences, 261 S. College Avenue, 111 Robinson Hall, Newark, DE 19716 \\ ${ }^{6}$ School of Development, Azim Premji University, PES Campus, Pixel Park, B Block, Electronics City, Hosur Road (Besides NICE Road), \\ Bengaluru 560 100, India \\ ${ }^{7}$ School of Advances International Studies, Johns Hopkins University, 1740 Massachusetts Avenue, NW, Washington, DC 20036
}

\begin{abstract}
Seasonal migration to cities is a common livelihood strategy for forest-fringe households in central India. Based on a previously collected household survey of 5000 villages across 500 forest-fringe villages in 32 districts of central India, we identify migration patterns over the last 5 years. Villages with seasonal workers are widely dispersed ( $75 \%$ of surveyed villages) and $81 \%$ of destination cities had reported COVID-19 cases at the beginning of the lockdown. Using a disease spread model to assess distancing strategies if returning migrants carry the virus to villages, we find that lenient restrictions for people within a village combined with maximal restrictions between villages could reduce the number of people exposed compared with moderate restrictions both within and between villages. Such a 'village bubble' strategy could reduce the risk of spread among vulnerable populations and requires that essential goods reach villages.
\end{abstract}

Keywords: COVID-19, containment, forest-fringe villages, lockdown, migration.

SEASONAL migration to cities is a widespread livelihood strategy for poor rural households in India. In contrast to permanent migration, a young adult in the household migrates to a city where labour jobs are available. The migrant spends up to six months a year in the city and returns to the village, generally in time for the monsoon planting season. Seasonal migrants are generally poorer, less educated and own less land than other types of migrants who move to cities permanently for employment or for educational opportunities ${ }^{1}$. With the outbreak of COVID-19, the potential for seasonal migrants to carry the virus from cities to their home villages generates

*For correspondence. (e-mail: rd2402@columbia.edu) grave concern both for local people and authorities managing the crisis.

Households living near forests are among the poorest and most vulnerable populations in India. Central India is one of the main forested areas in the country, with high proportion of Scheduled Tribe (ST) populations in forestfringe villages. The poorest households in these villages use seasonal migration to supplement their incomes, raising the potential for exposure to COVID-19 as migrants return. Many other factors increase the vulnerability of these populations, including poor availability and access to health facilities, crowded households, low-quality $\operatorname{diets}^{2}$, and a high proportion of households using fuelwood which generates indoor air pollution and compromised respiratory health. While the relatively low population density of rural areas relative to cities reduces the chances of COVID-19 spreading to a large number of people, the impact of exposure on vulnerable populations and spread between forest-fringe villages of Central India is severe.

Travel for migrants living in cities became difficult after the 21-day national lockdown, which began on 24 March 2020 and was extended until at least 3 May 2020. News reports show migrants returning to their villages on foot and by any means possible. Quarantine facilities in both cities and villages aim to restrict transmission in villages, but the effectiveness of these efforts is unknown. The government restricted inter-state travel for migrants after 20 April 2020. As restrictions ease, chances of exposure and spread to adjacent villages from migrants who have already returned persist. Alternatives to severe physical distancing are needed to reduce the chances of exposure while allowing people to obtain essential supplies, plant crops and carry out other necessities of daily life. 


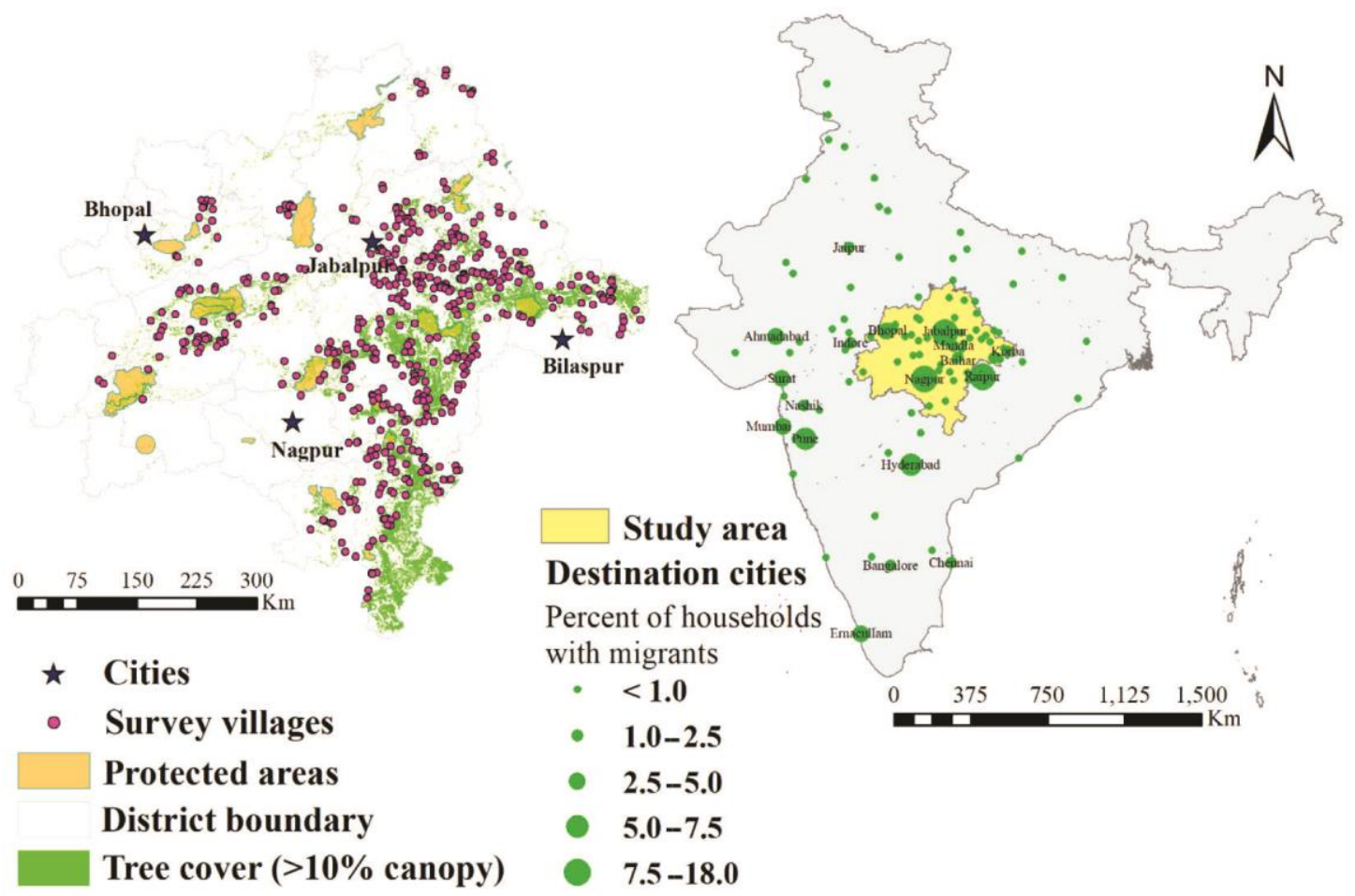

Figure 1. Locations of surveyed villages (left) and destinations (right) for forest-fringe villages in Central India. Tree cover data from Hansen $e t$ al. $^{13}$.

During January to March 2018, we surveyed 5000 households in approximately 500 forest-fringe villages in Central India to assess patterns of migration over the previous five years. The purpose of the survey was unrelated to disease spread. However, the patterns of migration identified from these surveys become relevant as the spread of COVID-19 due to migrants travelling from cities to rural areas gains national attention.

In this study, we use these survey results to address two questions: to what extent and where are forest-fringe villages in Central India potentially vulnerable to exposure from returning seasonal workers based on migration patterns over the last five years; and which physical distancing strategies (movement restrictions within and between villages) could be effective in reducing the likelihood of spread among adjacent villages as authorities ease distancing restrictions.

\section{Data and methods}

\section{Study region}

We define the Central Indian region to include 32 administrative districts spanning three states - Madhya Pradesh, Chhattisgarh and Maharashtra with a population of 54 million people (Figure 1). It covers approximately $7.6 \%$ of total land area in India (approximately $25 \mathrm{~m} \mathrm{ha}$ ) and contains some of the largest remaining forest patches in the country ${ }^{3}$. Seventy per cent of the population is rural. Livelihoods of the rural population consist of small-scale farming, livestock rearing and collection of forest products (Baquie, S. et al., pers. commun.). The populations include many tribal communities living in and around forests, with Gonds and Baigas as the main tribes. Twentytwo per cent of the population is recorded as ST compared with approximately $9 \%$ nationally. Availability of health facilities is more than five times less than the national average. Eighty-seven per cent of rural households rely on wood as a primary cooking fuel, although recently, the availability of LPG has penetrated into remote areas. Five of the districts are included as 'aspirational districts' in the government's programme for improving nutrition, health, education and other dimensions ${ }^{4,5}$ (Supplementary Table 1).

Approximately $37 \%$ of all villages in the study region are within the forest fringe (defined here as within $8 \mathrm{~km}$ of a forest patch greater than $500 \mathrm{ha}$ ), with the percentage varying across districts from as low as 9 to as high as 91 (Supplementary Table 1). Those living in the forest fringe depend on forests for fuelwood, timber, fodder and nontimber forest products. Weekly markets sell vegetables and other food items. People walk or use crowded autorickshaws to travel to weekly markets, which draw villagers from catchment areas of multiple villages. People also travel to small towns and district centres to purchase agricultural inputs and conduct paperwork. 
For those villages in the forest fringe, the median distance to the nearest neighbouring village is $1.7 \mathrm{~km}$. Median population size of forest-fringe villages (2011 Census) is 570 people and 127 households (Supplementary Figure 1). The small village populations and close proximity to neighbouring villages suggest that interactions between people from different villages can frequently occur.

\section{Household survey}

We conducted household surveys from January to March 2018, across approximately 500 forest-fringe villages and 5000 households (10 households per village). We divided villages within $8 \mathrm{~km}$ of the nearest forest edge into four groups split by median distance to road and town, and then randomly sampled 125 villages from each of the four groups. Each surveyed household responded to an approximately 45 min interview, which included questions about the number of people in the household who had migrated in the last five years, duration and destination of migration, year of initial migration and reasons for migration. We also carried out a village-level survey with village representatives to enquire about households whose members have left the village permanently.

Previous analyses of survey results indicate that the overwhelming majority of migration in this region is seasonal with less than $1 \%$ of households migrating permanently. A total of 889 (18\%) of 5000 households had a person working outside the village. In addition, households with seasonal migrants were poorer and more likely to have no income source in at least one season compared with households without members who migrate. About half the migrants stayed away from home for more than three months but returned to the village each year, and half stayed away less than three months. Approximately $45 \%$ of migrants went to destinations within the study region $^{6}$.

\section{Analysis of household surveys for migration patterns}

As an indication of whether authorities could expect COVID-19 in particular clusters in a region, we assess how widely the households with migrating members are dispersed across the region. We determine the proportion of districts, villages within districts and households within villages which potentially could be exposed to COVID-19 from returning migrants. Based on reports of the destination city for migrants, we use the publicly available COVID-19 tracker site (www.covid19india.org) for the number of reported cases in each district in which the city is located. We recorded the reported cases on 29 March (five days after the beginning of the lockdown) and, for comparison, on 16 April. We expect that cases are underreported and so use these values only to indicate where COVID-19 might have been present on those dates.

\section{Disease spread model}

We use an epidemiological deterministic SEIR compartmental model to test the hypothetical spread of COVID19 within and between adjacent villages with one of the villages exposed to the virus ${ }^{7}$. The model estimates the flow of individuals between four states: susceptible (S), exposed but not infectious during the incubation period (E), infected (I) and recovered (R). We parameterize the model for an average village of those included in the survey, using the median population and values from available reports for the duration of exposed and infected states. Initial values for susceptible population is 712 individuals, one exposed and no recovered individuals, duration of incubation period as six days based on Klein et $a l .{ }^{8}$, duration of infectivity period as 14 days based on Lauer et al. ${ }^{9}$, and case fatality rate of 0.02 , based on the value for India (www.worldometers.info/coronavirus/ \#countries). For a village with migrants and a population of 712 individuals, approximately 25 can be expected to be migrants based on median household size of five people and $18 \%$ of households with migrants (see Results). However, because we do not know how many migrants are actually exposed, the model assumes one exposed individual. We also run the model with alternative values for durations of incubation and infectivity periods to test sensitivity to these parameters.

We run the model for 500 time steps (days) (a long enough time for flow from exposed to susceptible to reach zero) with a range of values for $\mathrm{R} 0$, the basic reproduction number that represents the expected number of cases generated by an infected individual. R0 describes the average transmissibility of an infectious disease, which reflects the inherent contagiousness of the pathogen and the number of contacts for an individual. We vary R0 to represent leniency or strictness of physical distancing between individuals in the case of one village with exposure to COVID-19 that is adjacent to another village without exposure. We chose the upper bound as 3.0 based on Klein et al. ${ }^{8}$ and the lower bound as 1.0 to reflect the value below which the spread will die out.

To compare possible physical distancing strategies as restrictions ease after the lockdown, we run the SEIR model varying the $\mathrm{R} 0$ value to reflect the effectiveness of movement restrictions within a village and between adjacent villages. The goal is to identify the strategy with the lowest proportion of people exposed in the two villages combined. We calculate the proportion of exposed people relative to a baseline of no movement restrictions $(\mathrm{R} 0=3.0)$ for different combinations of lenient and maximal movement restrictions within and between villages (Figure 2). We use the R0 values 1.0, 2.0 and 3.0 to 
represent maximal, moderate and lenient movement restrictions respectively. Maximal restriction $(\mathrm{R} 0=1.0)$ suggests that contact between individuals would occur at very low levels as during the strict lockdown period when people were instructed to stay at home. Lenient movement $(\mathrm{R} 0=3.0)$ suggests contact rates that occurred prior to the lockdown. Moderate movement restrictions $(\mathrm{R} 0=2.0)$ suggest midway between maximal and lenient restrictions, with contact rates about half of those that would normally occur, which could arise from limited hours for shops and reduced number of people in buses.

To estimate the proportion of the population exposed, we use the combined population of the two villages (1424 people). For example, a scenario of maximal movement restrictions between villages and lenient restrictions within villages is calculated from the proportion of people exposed with $\mathrm{R} 0=3.0$ for 712 susceptible individuals (relative to 1424 individuals). A scenario of moderate movement restrictions within and between villages is the proportion of individuals exposed with R0 $=2.0$ for 1424 susceptible individuals.

For each model run, we calculate the total number of exposed people as the sum of the flow from susceptible to exposed over the 500-day time period. We estimate the number of exposed people without stratifying by age or

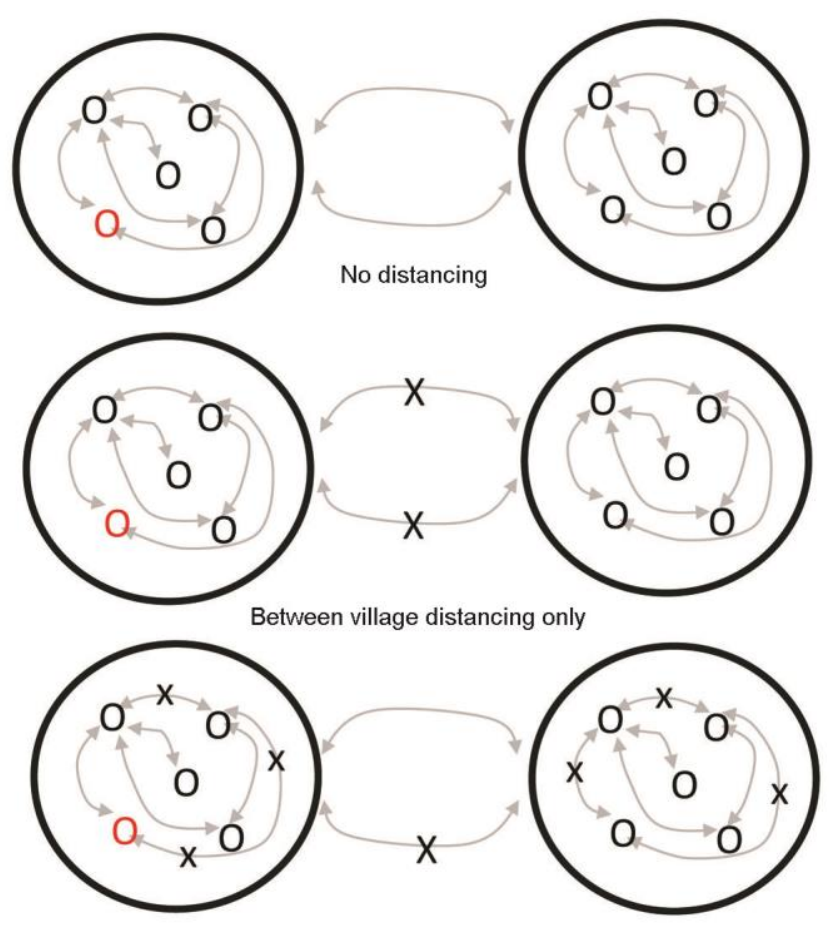

Moderate within and between village distancing

Figure 2. Conceptual physical distancing strategies between adjacent villages: (top) no restrictions, (middle) lenient within village and maximal movement restrictions between villages, and (bottom) moderate movement restrictions between and within villages. $\mathrm{O}$ represents individuals with red indicating one exposed individual, $\mathrm{X}$ represents physical distancing between individuals and large circles represent villages. attempting to estimate the number of actual COVID-19 cases. The values used to parametrize the model are intended to compare the relative effectiveness of different physical distancing strategies, rather than to estimate the actual number of people who would display symptoms of COVID-19 or require health facilities. We use the $\mathrm{R}$ package EpiModel ${ }^{7}$ with code modified for an SEIR model, as provided in http://statnet.org/tut/NewDCMs. html.

The model represents an over-simplified, stylized case of two adjacent villages, one with an exposed individual and one with no exposed individuals (note that the actual likelihood that a returning migrant is far less than one depends on the probability that a migrant was actually exposed). The model assumes uniform mixing between the two villages (in the case of lenient and moderate movement restrictions) and complete isolation in the case of maximal movement restrictions between villages.

\section{Results}

\section{Migration patterns across forest-fringe villages}

We find that seasonal migration from villages is widely dispersed across forest-fringe villages in Central India. Also, 371 out of $495(75 \%)$ of villages had at least one household using seasonal migration for at least one member as a livelihood strategy (Figure $3 a$ ). On an average for a village with migrants, $2.38( \pm 0.02$ for $95 \%$ confidence interval) out of ten surveyed households in the village had a member migrating in the last five years. All 32 districts had at least one surveyed village with at least one household using that strategy, with $34 \%$ (11 out of 32 districts) having more than $90 \%$ of surveyed villages with seasonal migrants (Figure $3 b$ ). Supplementary Table 2 provides these proportions by district (note that some districts have only a small number of surveyed villages and so extrapolation is not reliable). Destination cities for migrants were also widely dispersed, without a pattern of migrants from particular locations travelling to particular places. In the last five years, seasonal migrants went to 124 destinations. Many of these destinations are smaller cities and towns, with $18 \%$ of the migrants going to 82 destinations with population less than a million people. Some destinations are larger cities, predominantly Nagpur (18\% of migrants), Jabalpur (14\%), Raipur (9\%), Pune $(6 \%)$ and Hyderabad $(6 \%)$, where there is higher likelihood of COVID-19 cases (Supplementary Table 3). Thirty-seven per cent of the 124 destinations had at least one reported COVID-19 case on 29 March 2020, to which $81 \%$ of the migrants went. By 16 April 2020, 65\% of the destinations had reported COVID-19 cases, these were the destinations of $89 \%$ of the seasonal migrants. Reported cases increased over 16-fold between the two dates (Figure 4). Note that these destinations are reported 

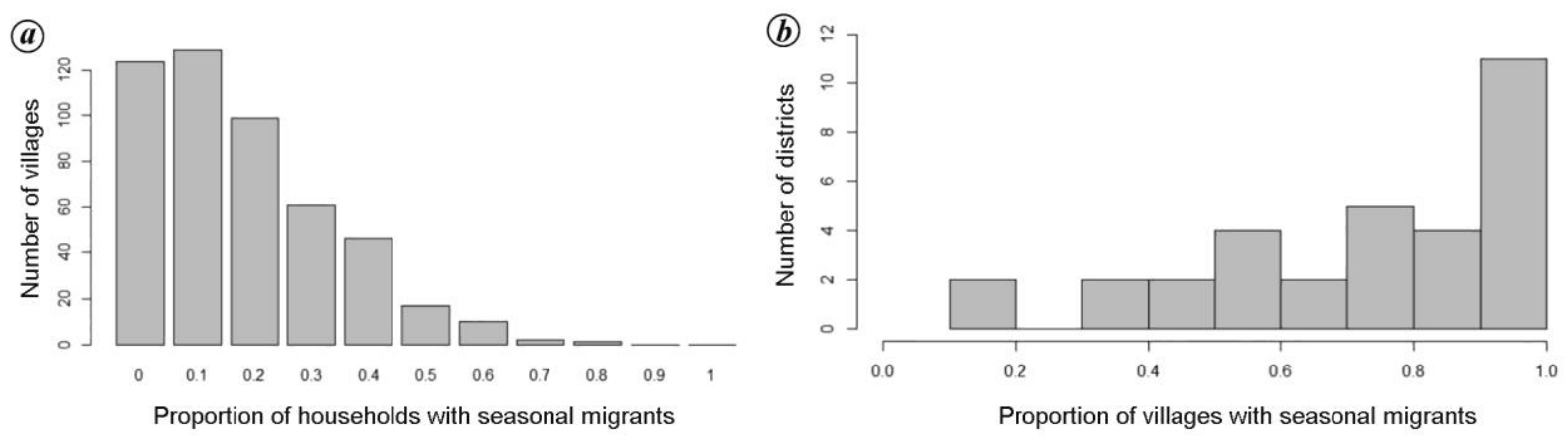

Figure 3. (a) Number of villages with varying proportions of surveyed households with seasonal migrants and (b) number of districts with varying proportions of surveyed villages with seasonal migrants from forest-fringe villages in Central India. See Supplementary Table $\underline{2}$ for district-wise proportions.
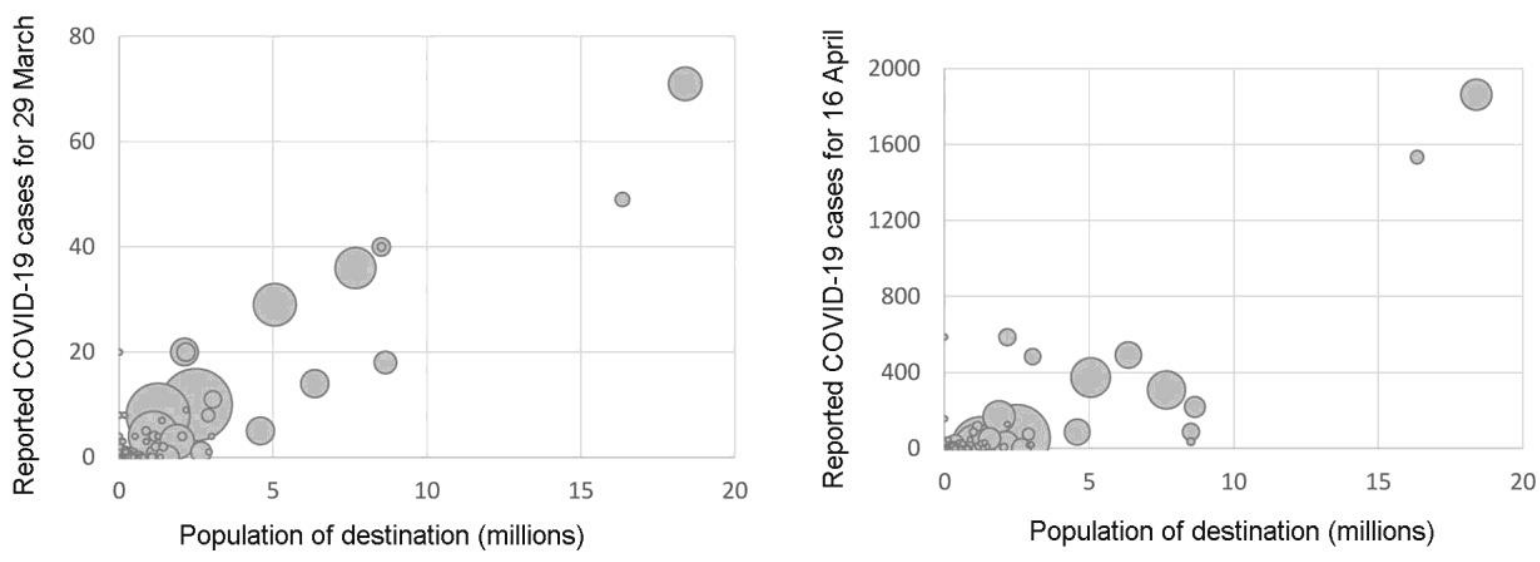

Figure 4. Reported cases in destination locations for seasonal migrants in Central India for 29 March and 16 April 2020. Size of the bubble is proportional to the proportion on total migrants reaching the destination. Note difference scale for the two dates. Population is from the 2011 Census ${ }^{14}$ using values for urban agglomerations.

for the last five years and do not necessarily represent those during the COVID-19 outbreak, though one can expect similar patterns.

Based on these representative proportions, the probability that at least one migrant returns from a destination where COVID-19 has been reported, for an average village with 25 migrants returning, is close to one (1-(1$\left.0.81)^{\wedge} 25\right)$. This probability represents only potential transmission and the actual probability of a returning migrant carrying the virus to a village. The actual probability would be much smaller depending on the proportion of migrants who carry the virus from a city to a village, which could depend on the number of cases and size of the city, living conditions, whether the migrant was actually in the city at the time of the COVID-19 outbreak, whether the migrant was quarantined before or after returning to the village, and other variables. The widely dispersed geography of both village origins and city destinations for seasonal migrants in the region, which results in potential exposure across forest-fringe villages in the region, indicates the need for practicable strategies to minimize chances of spread as lockdown restrictions ease.

\section{Effectiveness of strategies for movement restrictions within and between villages}

Results from the SEIR model show the strong sensitivity of the number of exposed people to the R0 value. In the case of two adjacent villages, with one person in one of the villages carrying the virus, the question is whether a more effective strategy is to keep maximal movement restrictions between villages while allowing interaction within villages, or to keep moderate movement restrictions between individuals both within and between villages. As the number of exposed people is highly sensitive to the R0 value (Figure 5), the question is most relevant at high values. With the high transmissibility of the coronavirus (inherently high R0) and severe economic hardships of physical distancing, the maximum benefit in terms of people exposed for the minimum movement restrictions would be the most beneficial strategy as restrictions ease.

Results from the SEIR model provide a conceptual picture of the effectiveness of different strategies for graduated easing of restrictions following the lockdown period, in terms of proportion of population exposed. Maximal 
movement restrictions both between and within villages (top scenario in Figure 2) exposes only $2 \%$ of the population and lenient distancing both between and within villages exposes 94\% (Table 1). If restrictions ease to allow lenient distancing within villages $(\mathrm{R} 0=3.0)$ but maintain maximal movement restrictions between villages (middle scenario in Figure 2), the model estimates that $47 \%$ of the population of both villages would be exposed. For a scenario of moderate restrictions both between and within villages (bottom scenario in Figure 2), more than $80 \%$ of people are exposed. To achieve the same level of exposure as the middle scenario (47\%) with mixing both between and within villages, the R0 value would need to decrease by more than half, from 3.0 to approximately 1.3 (red line in Figure 5). Varying the input parameters in the model for exposed and susceptible number of individuals, and for duration of incubation and infectivity periods alters the result only slightly with the same overall conclusion (Supplementary Table 4). Incidentally, reports from elsewhere in the country indicate that villagers are implementing a strategy of movement restriction between villages through blockages to entries and exits ${ }^{10}$.

In summary, a post-lockdown strategy to maintain movement restrictions between villages while easing restrictions within villages could result in fewer number of people exposed than moderate easing of restrictions both within and between villages.

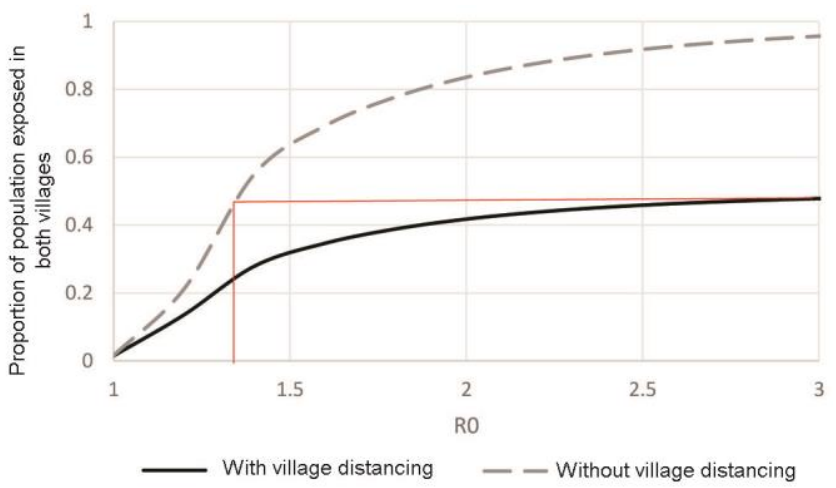

Figure 5. Proportion of total population exposed in two hypothetical villages, with one exposed individual in one of the villages, with and without movement restrictions between the villages. The red line indicates R0 value required (approximately 1.3) to achieve the same proportion of population exposed with physical distancing within and between villages as with no within village but between village distancing.

Table 1. Proportion of total population exposed in two villages for scenarios of movement restrictions between and within villages for maximal $(\mathrm{R} 0=1)$, moderate $(\mathrm{R} 0=2)$ and lenient $(\mathrm{R} 0=3)$ distancing

\begin{tabular}{lcc}
\hline Between villages & Within a village & Proportion exposed \\
\hline Maximal & Maximal & 0.02 \\
Lenient & Lenient & 0.94 \\
Maximal & Lenient & 0.47 \\
Moderate & Moderate & 0.80 \\
\hline
\end{tabular}

\section{Discussion and conclusion}

The spatial distribution of seasonal migration across forest-fringe areas of Central India paints a picture of widespread potential for exposure to COVID-19. These populations are among the most vulnerable in the country with the potential for extreme hardship if COVID-19 spreads.

Although the proportion of villages with migrants varies across districts, migration patterns do not indicate concentrated hotspots of either origins (villages) or destinations (cities). The number of migrants who have already returned to villages prior to or during the lockdown is unknown. These patterns suggest that authorities need to prepare for the possibility of outbreaks throughout the forested areas of the region. Humane and nonstigmatizing facilities to quarantine people, as well as testing and health facilities, are needed with flexibility to respond to an outbreak where it might occur.

Some districts in the region have particularly high proportions of forest-fringe villages with migrants as well as high proportions of households within villages with migrants. These villages are particularly susceptible to the spread of the virus. Authorities could focus particular attention on testing and preparation of health facilities in these areas using multiple metrics such as those in Table 1. Dogra et al. ${ }^{11}$ provide a framework to assess vulnerability.

As restrictions ease to allow critical activities such as collecting fuelwood, selling and shopping at village markets, purchasing and planting seeds, and other activities, practical physical distancing and movement restrictions will still be required. A primary concern is to contain the spread of the virus across villages in the case of exposure in one village. To reduce chances of exposure in the population in a rural setting such as Central India, the model results suggest that a prudent post-lockdown strategy might focus on easing physical distancing restrictions within villages but maintaining movement restrictions between villages, while quickly imposing strict lockdown both within and between villages if a case emerges. Such a 'village bubble' strategy would allow people to interact within case-free villages, but would require availability of food, energy, agricultural inputs and other essentials without the need to travel to other villages or towns. Because many vulnerable communities in forest-fringe villages are food-insecure, the ability to acquire essentials while minimizing risks of exposure to COVID-19 takes on added urgency.

A 'village bubble' strategy might be applicable in other rural settings throughout India and is reportedly being implemented instinctively at the village level. Interestingly, New Zealand has successfully implemented a bubble strategy, with initial bubbles restricted to the household level and broadened to larger social groups as restrictions ease $^{12}$. 
This analysis has limitations based on the hypothetical nature of the model assumptions. The simplified modelling assumptions regarding complete mixing or isolation between villages provides a conceptual basis for comparing alternative distancing strategies in the absence of empirical data. Testing and monitoring the effectiveness of different strategies at the local level are needed to protect the vulnerable populations in forest-fringe villages of Central India.

1. Srivastava, R., Internal migration in India: an overview of its features, trends and policy challenges. Social and Human Sciences Sector, UNICEF, 2011, 6-7.

2. Neelakantan, A. et al., Contributions of financial, social and natural capital to food security around Kanha National Park. Reg. Environ. Change, 2020. 20(1), 1-14.

3. Nayak, R., et al., Bits and pieces: impact of linear institutions on forest structural connectivity in India. Land Use Policy, 2020, 104619.

4. NITI Aayog, Deep dive: insights from champions of change, the aspirational districts dashboard. NITI Aayog, New Delhi, 2018.

5. Alkire, S., Oldiges, C. and Kanagaratnam, U., Multidimensional poverty reduction in India 2005/6-2015/16: still a long way to go but the poorest are catching up, Oxford Poverty and Human Development Initiative (OPHI), OPHI Research in Progress Series, 2018, 54A, 1-30.

6. Velho, N. et al., Impacts of Urban Migration on Forest use in Central India (eds Mundoli, S. and Nagendra, H.), Azim Premji University, Bengaluru, India.
7. Jenness, S. M., Goodreau, S. M. and Morris, M., EpiModel: an R package for mathematical modeling of infectious disease over networks. J. Stat. Software, 2018, 84, 1-47.

8. Klein, E. et al., Covid-19 for India update. Center for Disease Dynamics, Economics, and Policy and Johns Hopkins University, USA, 2020.

9. Lauer, S. A. et al., The incubation period of coronavirus disease 2019 (COVID-19) from publicly reported confirmed cases: estimation and application. Ann. Int. Med., 2020.

10. Anon, Villages abide by lockdown, fence entry and exit. The Times of India, 1 April 2020.

11. Dogra, N. et al., Pilot tool for assessment of health vulnerability to climate change at the sub-national level in India. International Institute of Health Management Research, New Delhi, India, 2013.

12. Olmstead, M., New Zealand's 'bubble concept' is slowly letting people socialize again. Would it work in America?. Slate, 2020.

13. Hansen, M. C. et al., High-resolution global maps of 21 st-century forest cover change. Science, 2013. 342(6160), 850-853.

14. Registrar General and Census Commissioner, Census of India 2011. Office of the Registrar General, New Delhi, 2011.

ACKNOWLEDGEMENTS. The survey was conducted under IRB Protocol Number: IRB-AAAR5819 and funded by NASA Land Use and Land Cover Change program grant number NNX17AI24G for the project 'Tropical Deciduous Forests of South Asia: Monitoring Degradation and Assessing Impacts of Urbanization'. We thank to Nandini Velho for assistance with collection and analysis of the survey data, and Maria Diuk-Wasser and Jeff Shaman for advice on epidemiological models.

Received 28 April 2020; revised accepted 18 May 2020

doi: $10.18520 / \mathrm{cs} / \mathrm{v} 119 / \mathrm{i} 1 / 52-58$ 